\title{
THE ASSOCIATION OF OLFACTORY FUNCTION WITH BMI, APPETITE, AND PROSPECTIVE WEIGHT CHANGE IN DUTCH COMMUNITY-DWELLING OLDER ADULTS
}

\author{
K.S. FLUITMAN ${ }^{1,2}$, H.J. NADAR ${ }^{3}$, D.S. ROOS ${ }^{4}$, H.W. BERENDSE ${ }^{4}$, B.J.F. KEIJSER ${ }^{5,6}$, M. NIEUWDORP ${ }^{1,7}$, \\ R.G. IJZERMAN ${ }^{1}$, M. VISSER ${ }^{3}$
}

\begin{abstract}
1. Department of Internal Medicine, Amsterdam UMC, Vrije Universiteit Amsterdam, Amsterdam Public Health Research Institute, Amsterdam, The Netherlands; 2. Wallenburg Laboratory, Department of Molecular and Clinical Medicine, Sahlgrenska Academy, University of Gothenburg, Gothenburg, Sweden; 3. Department of Health Sciences, Faculty of Science, Vrije Universiteit Amsterdam, Amsterdam Public Health Research Institute, Amsterdam, The Netherlands; 4. Department of Neurology, Amsterdam UMC, Vrije Universiteit Amsterdam, Amsterdam, The Netherlands; 5. Department of Microbiology and Systems biology, TNO earth, Life and Social Sciences, Zeist, The Netherlands; 6. Department of Preventive Dentistry, Academic Center for Dentistry Amsterdam, University of Amsterdam and Vrije Universiteit Amsterdam, Amsterdam, The Netherlands; 7. Department of Vascular Medicine, Amsterdam UMC, University of Amsterdam, Amsterdam, The Netherlands. Corresponding author: Drs. K. S. Fluitman, Department of Internal Medicine, Amsterdam UMC, Vrije Universiteit Amsterdam, De Boelelaan 1117, 1081 HV Amsterdam, The Netherlands, E-mail: k.fluitman@vumc.nl, Tel: +31(20)4440651
\end{abstract}

\begin{abstract}
Objectives: The olfactory decline that often accompanies aging is thought to contribute to undernutrition in older adults. It is believed to negatively affect eating pleasure, appetite, food intake and subsequently nutritional status. We have evaluated the associations of olfactory function with BMI, appetite and prospective weight change in a cohort of Dutch community-dwelling older adults. Design: Cross-sectional cohort study. Participants: Dutch community-dwelling older adults from the ongoing Longitudinal Aging Study Amsterdam (LASA). Measurements and setting: In 2012-2013, the 40-item University of Pennsylvania Smell Identification Test (UPSIT) was administered to 824 LASA participants to evaluate their olfactory function. Body weight, height, appetite, comorbidity, cognitive status and socio-demographic factors were also assessed. Follow-up weight was measured after three years. Results: 673 participants (aged 55-65 years) were included in the regression analyses. Median UPSIT-score was 33. When adjusted for potential confounders, lower UPSITscore (indicative of poorer olfactory function) was not associated with poor appetite (OR $=1.062, \mathrm{p}=0.137$ ) or prospective weight change $(\mathrm{B}=-0.027, \mathrm{p}=0.548)$. It was, however, associated with lower $\mathrm{BMI}$ in smokers $(\mathrm{B}=0.178, \mathrm{p}=0.032)$, but not in non-smokers $(\mathrm{B}=-0.015, \mathrm{p}=0.732)$. Conclusion: Lower olfactory function scores were associated with lower BMI in community-dwelling older adults who smoke, but not with appetite or prospective weight change. Therefore, smoking older adults with olfactory impairments may pose as a vulnerable group with respect to developing undernutrition.
\end{abstract}

Key words: Olfactory function, BMI, appetite, weight, older adults.

\section{Introduction}

An important health concern in the older population is protein-energy undernutrition (1). This is a form of malnutrition frequently resulting from insufficient energy or protein uptake (2). Its prevalence rates vary from $7-16 \%$ in communitydwelling older adults to $18-33 \%$ in institutionalized older adults (3). However, since about $95 \%$ of the Dutch older population lives at home, this is where most cases reside (4).

In order to develop effective preventive interventions, a wide interest in the determinants of undernutrition in older adults has emerged (5). The age-related decline in olfactory function (presbyosmia) is argued to be one of these determinants $(6,7)$. Presbyosmia affects up to $60 \%$ of the aged population (8-11). It is hypothesized to cause appetite suppression and reduce food intake, increasing the risk of developing undernutrition $(12,13)$. Nevertheless, the potential importance of olfaction in relation to healthy ageing is underappreciated in comparison with other senses, such as hearing and vision (14). Also, current literature on the relationship between olfaction and nutritional status is inconsistent $(11,14-20)$. In a large cohort of 1636 older adults aged over 60 , poorer olfactory function was indeed associated with lower BMI (20). In a sub-sample of 557 older adults from this cohort, Gopinath et al. found that women with olfactory impairment at baseline had lower dietary quality 5 years later (14). In addition, Seubert et al. studied 2234 older adults and also found olfactory dysfunction to be associated with both a $\mathrm{BMI}<18$ and poor appetite (11). Conversely, it has been reported that olfactory dysfunction was not at all related to diminished eating pleasure or poor appetite (17) or to risk of malnutrition, measured by either low BMI or low MiniNutritional Assessment score $(16,18,19)$. Aside from the study by Gopinath et al. (14) these studies were all cross-sectional $(15-20)$.

In the current study, we aimed to evaluate the relationship between impaired olfactory function (measured with an extensive 40-item smell identification test), poor appetite and low BMI in a large cohort of 824 community-dwelling Dutch older adults. In addition, we tested whether impaired olfactory function is associated with 3-year prospective weight change. If olfactory dysfunction indeed contributes to poor appetite and the development of undernutrition, dietary interventions should specifically be tailored to those older adults with an impaired sense of smell (12). 


\section{THE JOURNAL OF NUTRITION, HEALTH \& AGINGC}

\section{Materials and methods}

\section{Subjects}

Data were used from the ongoing, large, multidisciplinary Longitudinal Aging Study Amsterdam (LASA) (21). The main objective of LASA is to examine the predictors and consequences of ageing in community-dwelling Dutch older adults. This is described more elaborately elsewhere $(21,22)$.

In 2012-2013, a third LASA-cohort aged 55-65 years was included $(\mathrm{n}=1023)$. These 1023 participants were asked to undergo an additional olfactory test. Of them, 199 declined and 824 consented. The study was approved by the local medical ethics review board.

\section{Olfactory function}

Olfactory function was measured by the self-administrated University of Pennsylvania Smell Identification Test (UPSIT) (23). The UPSIT-test consists of 40 different microencapsulated odors, which are released by scratching the microcapsule. Participants are then required to identify each odor by choosing from four forced-choice response options. Participants were also asked if they experienced any smell problems in general, with answering categories 'yes' and 'no'. Since the UPSITscore is gender dependent, the following olfactory function categories were used as provided by the test manufacturer: normosmic (34-40 for male and 35-40 for female), microsmic (19-33 for male and 19-34 for female) and anosmic (<19 for both male and female) (24).

\section{Anthropometry}

Body weight $(\mathrm{kg})$ and height $(\mathrm{m})$ were measured during the LASA interviews by trained interviewers. Measured weight was adjusted by subtracting $1 \mathrm{~kg}$ if the participant was wearing shoes, clothes, or a corset. In case of unavailable measurements, self-reported body weight or height were used. BMI $(\mathrm{kg} / \mathrm{m} 2)$ was calculated. Weight change was assessed by subtracting baseline weight from follow-up weight measurement in 20152016 (3-year follow-up).

\section{Appetite}

Information on appetite was obtained using the second question from the Dutch translation of the Centre for Epidemiologic Studies Depression scale (CES-D) (25): "In the past week, I did not feel like eating, my appetite was poor". Possible answers were: 1 "rarely or never", 2 "some of the time", 3 "often", and 4 "most of the time or always". Appetite was dichotomized as follows: no problems with appetite (answer 1) and poor appetite (answers 2-4) (26).

\section{Assessment of covariates}

Information on socio-demographics (age, sex and education), lifestyle factors (alcohol use and smoking status), co-morbidity (number of chronic diseases, medication use and depression), and cognitive status were all collected by questionnaires.
Education was defined as high (higher vocational, college or university education), medium (general secondary education, lower and intermediate vocational education, and general intermediate education) or low (elementary education completed or not completed). The Garretsen alcohol consumption index was used to categorize alcohol consumption into four categories based on number of times drinking per month and number of drinks consumed each time: excessive, moderate, light, and no alcohol use (27). Smoking status was dichotomized into non-smokers (never or former smoker) and current smoker. For current smokers, the number of cigarettes per week was documented. Three categories were created the number of chronic diseases: no chronic diseases, one chronic disease, and two or more chronic diseases. Medication use was assessed by asking the respondents to show their prescribed medication containers to the interviewers. It was classified into three categories: no medication use, use of one to four medications, and use of five or more medications. Depressive symptoms were dichotomized using the 20-item CES-D scale. A CES-D score above 15 out of 60 indicated depression (25). Cognitive status was measured by the Mini Mental State Exam (MMSE) with a score ranging from 0 to 30. A score of 23 or less was indicative of impaired cognitive function (28).

\section{Statistical analysis}

Participant characteristics are depicted by means \pm standard deviations (SD) or medians and interquartile ranges (IQR) for continuous variables. Categorical characteristics are depicted by numbers and percentages (\%). Differences among the olfactory categories (normosmic, microsmic and anosmic) were tested using ANOVA or Kruskal-Wallis test (continuous variables) and Fisher's Exact test (categorical variables).

For the main analyses, the UPSIT-score was used as continuous independent variable in regression models. Linear regression analyses were performed to analyze the associations of UPSIT-score with BMI and of UPSIT-score with prospective weight change. First, crude models were made, which were checked for potential effect modifiers: sex, age, smoking status, and appetite. In case of significant effect modification $(\mathrm{p}<0.10)$, the data were stratified accordingly. Each model was then adjusted for potential confounders including: education, smoking, alcohol use, number of medications and chronic diseases, MMSE-score, and CESD-score.

The association between olfactory function and appetite was examined with logistic regression. Effect modification and confounding were examined as described above. In order to use CES-D score as covariate in the logistic regression, the appetite question, which was also the outcome measurement, was excluded from calculating the CES-D score.

A two-tailed $\mathrm{p}$ value of $<0.05$ was considered statistically significant. All statistical analyses were performed using SPSS software version 22 (SPSS Inc., Chicago Illinois). 


\section{Results}

\section{Participants' characteristics}

Of the 824 LASA-participants who had consented to the UPSIT-test, 673 completed all UPSIT-questions and were included in the analyses. Figure 1 depicts the flowchart of all participants.

\section{Figure 1}

Flowchart of number of participants of the Longitudinal Aging Study Amsterdam (LASA) included in the final analyses

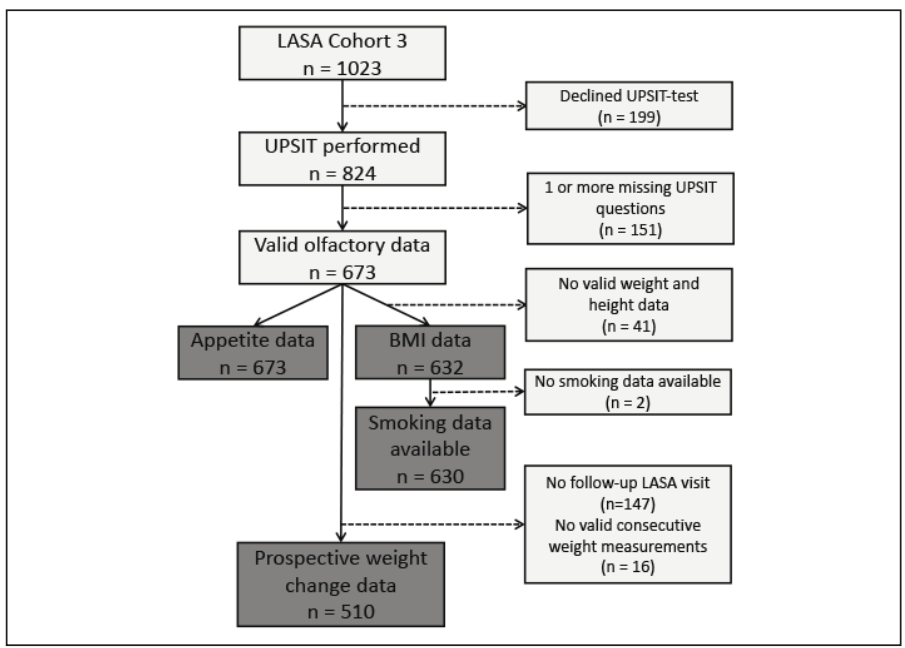

LASA: Longitudinal Aging Study Amsterdam; UPSIT: University of Pennsylvania Smell Identification Test; BMI: Body Mass Index.

The participant characteristics are shown in table 1 . The median (IQR) of the UPSIT-score was 33 (30-35). Based on their UPSIT-scores, 15 (2.2\%) participants were anosmic, 415 $(61.7 \%)$ were microsmic and $243(36.1 \%)$ were normosmic. The prevalence of self-reported smell difficulties was associated with the olfactory categories. However, about one-third (38.5\%) of the participants with functional anosmia did not report having any smelling problems (figure 2).

In our sample, subjects with microsmia and anosmia had a lower level of education: $72 \%$ of the microsmic group and $86.7 \%$ of the anosmic group had low or medium educational levels compared to $58.0 \%$ of the normosmic group. Also, participants with microsmia and anosmia were more likely to be current smokers (19.3\% and $13.3 \%$, respectively) than participants with normosmia $(8.4 \%)$. There was no difference in the number of cigarettes smoked per week among the smokers in the olfactory categories. For the baseline body weight, self-reported instead of measured weight was used in 5 cases $(0.8 \%)$. No self-reported weight needed to be used for followup measurements. Univariate comparison of BMI, appetite and weight change did not reveal significant differences among the three olfactory categories.
Figure 2

Self-reported smell difficulties across the three olfactory categories based on the University of Pennsylvania Smell Identification Test (UPSIT)

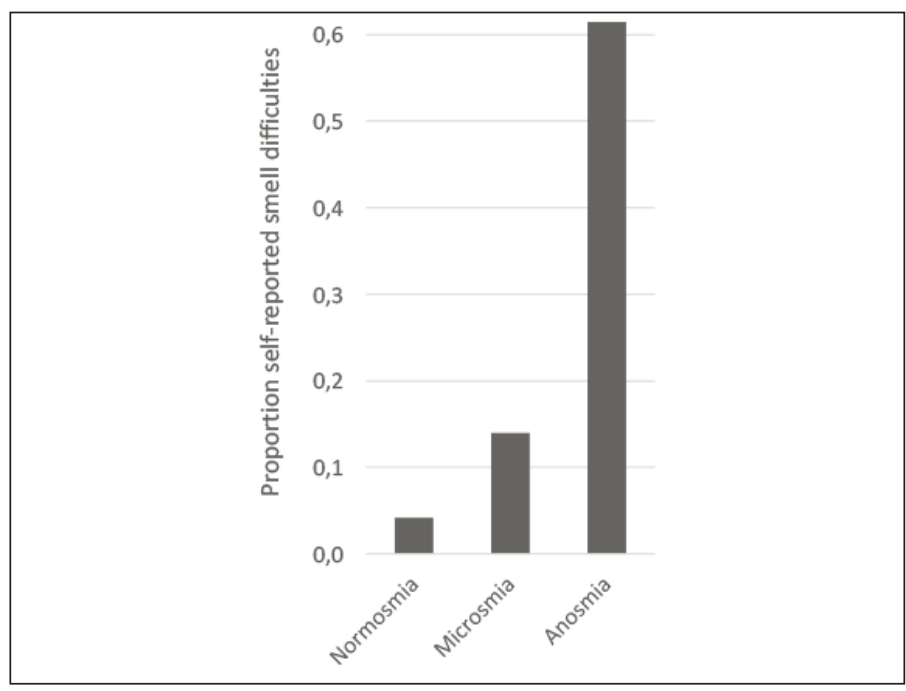

\section{Olfactory function and BMI}

The crude linear regression analysis revealed no significant association between UPSIT-score and BMI. Smoking status was found to be the only statistically significant effect modifier $(\mathrm{p}=0.009)$. In the smokers, lower UPSIT-score - indicating poorer olfactory function - was associated with lower BMI $(\mathrm{B}=0.225, \mathrm{SE}=0.078, \mathrm{p}=0.005$, table 2$)$. No such association was observed among non-smokers $(B=-0.042, \mathrm{SE}=0.045$, $\mathrm{p}=0.341$ ). The association of olfactory function with BMI in smokers remained statistically significant after adjusting for confounders $(\mathrm{B}=0.178, \mathrm{SE}=0.081, \mathrm{p}=0.032)$.

\section{Olfactory function and appetite}

Using binary logistic regression, no association was observed of UPSIT-score with poor appetite in the crude model $(\mathrm{OR}=1.029, \mathrm{p}=0.337)$, or the adjusted model $(\mathrm{OR}=1.062$, $\mathrm{p}=0.137)$. No effect modification was found.

\section{Olfactory function and prospective weight change}

Linear regression revealed no association of UPSITscore with 3-year weight change in the crude model $(B=-$ $0.033, \mathrm{SE}=0.044, \mathrm{p}=0.447)$ or the adjusted model $(\mathrm{B}=-0.027$, $\mathrm{SE}=0.045, \mathrm{p}=0.548)$. Both models were additionally adjusted for baseline weight and follow-up time in days. No significant effect modification by sex, age, smoking status, or appetite was found.

\section{Discussion}

Lower olfactory function scores were associated with lower BMI in older adults who smoke, but not in older adults who do not smoke. While the negative influence of smoking itself 
Table 1

Characteristics of the Longitudinal Aging Study Amsterdam (LASA) participants, stratified by olfactory function category based on the University of Pennsylvania Smell Identification Test (UPSIT)

\begin{tabular}{|c|c|c|c|c|c|}
\hline Characteristics & $\begin{array}{c}\text { All } \\
(n=673)\end{array}$ & $\begin{array}{c}\text { Normosmic } \\
(\mathrm{n}=\mathbf{2 4 3})\end{array}$ & $\begin{array}{c}\text { Microsmic } \\
(n=415)\end{array}$ & $\begin{array}{c}\text { Anosmic } \\
(\mathrm{n}=15)\end{array}$ & P-value* \\
\hline Age (years) & $60.4[57.8-63.1]$ & $60.4[57.7-63.1]$ & $60.3[57.8-63.1]$ & $60.9[58.2-63.9]$ & 0,79 \\
\hline Male & $328(48.7)$ & $122(50.2)$ & $201(48.4)$ & $5(33.3)$ & 0,451 \\
\hline BMI $(\mathrm{kg} / \mathrm{m} 2)(\mathrm{n}=632)$ & $27.0 \pm 4.6$ & $26.9 \pm 5.0$ & $27.2 \pm 4.4$ & $25.2 \pm 1.8$ & 0,228 \\
\hline Poor appetite & $69(10.3)$ & $23(9.5)$ & $46(11.1)$ & $0(0.0)$ & 0,465 \\
\hline 3 -year Weight change $(\mathrm{kg})(\mathrm{n}=510)$ & $-0.66 \pm 4.8$ & $-1.1 \pm 4.8$ & $-0.5 \pm 4.7$ & $1.6 \pm 5.0$ & 0,102 \\
\hline Follow-up time (days) $(\mathrm{n}=510)$ & $1134.6 \pm 59.4$ & $1139.4 \pm 62.3$ & $1131.6 \pm 57.2$ & $1128.8 \pm 66.7$ & 0,345 \\
\hline UPSIT-score & 33 [30-35] & $36[35-37]$ & 31 [29-33] & $16[12-17]$ & $<0.001 \%$ \\
\hline Self-reported smell problems $(\mathrm{n}=592)$ & $68(11.5)$ & $9(4.2)$ & $51(14.0)$ & $8(61.5)$ & $<0.001 \ddagger$ \\
\hline Current smoker $(\mathrm{n}=641)$ & $97(15.1)$ & $20(8.4)$ & $75(19.3)$ & $2(13.3)$ & 0.001 \$ \\
\hline Number of cigarettes/week $(n=84)$ & $75.0[29.8-109.3]$ & $56.0[19.5-101.3]$ & $84.0[35.0-118.5]$ & 56 & 0,185 \\
\hline \multicolumn{6}{|l|}{ Alcohol use $(n=642)$} \\
\hline No alcohol use & $80(12.5)$ & $25(10.5)$ & $52(13.3)$ & $3(20.0)$ & 0,129 \\
\hline Light & $262(40.8)$ & $96(40.5)$ & $157(40.3)$ & $9(60.0)$ & \\
\hline Moderate & $249(38.8)$ & $102(43.0)$ & $145(37.2)$ & $2(13.3)$ & \\
\hline (Very) Excessive & $51(7.9)$ & $14(5.9)$ & $36(9.2)$ & $1(6.7)$ & \\
\hline \multicolumn{6}{|l|}{ Number of chronic diseases } \\
\hline No chronic diseases & $285(42.3)$ & $106(43.6)$ & $172(41.4)$ & $7(46.7)$ & 0,939 \\
\hline 1 chronic disease & $260(38.6)$ & $94(38.7)$ & $160(38.6)$ & $6(40.0)$ & \\
\hline 2 or more chronic diseases & $128(19.0)$ & $43(17.7)$ & $83(20.0)$ & $2(13.3)$ & \\
\hline \multicolumn{6}{|l|}{ Number of medications $(\mathrm{n}=641)$} \\
\hline No medication use & $242(37.8)$ & $96(39.5)$ & $138(40.5)$ & $8(53.3)$ & 0,319 \\
\hline Use of 1 to 4 medications & $316(49.3)$ & $116(47.7)$ & $195(48.9)$ & $5(33.3)$ & \\
\hline Use of 5 or more medications & $83(12.9)$ & $25(10.3)$ & $56(17.7)$ & $2(13.3)$ & \\
\hline Poor cognitive status (MMSE $\leq 23$ ) & $11(1.6)$ & $4(1.6)$ & $6(1.4)$ & $1(6.7)$ & 0,307 \\
\hline $\begin{array}{l}\text { Depressive symptoms }(\mathrm{CESD} \geq 16) \\
(\mathrm{n}=671)\end{array}$ & 80 (11.9) & $23(9.5)$ & $55(13.3)$ & $2(13.3)$ & 0,292 \\
\hline \multicolumn{6}{|l|}{ Education } \\
\hline Low & $52(7.7)$ & $13(5.3)$ & $38(9.2)$ & $1(6.7)$ & 0.001 \$ \\
\hline Medium & $402(59.7)$ & $128(52.7)$ & $262(63.1)$ & $12(80.0)$ & \\
\hline High & $219(32.5)$ & $102(42.0)$ & 115 (27.7) & $2(13.3)$ & \\
\hline
\end{tabular}

on olfactory function has long been established, the effectmodifying role of smoking has not been reported before.

There are several possible explanations as to why poor olfactory function might be differently associated with BMI in smokers and non-smokers. Any association of olfactory function with BMI may normally be overshadowed by other factors that compensate for the loss of smell in older adults. For instance, an increased preference for specific food texture (like creamy foods) or taste (like salty and sugary foods) has been reported (15), and was suggested to secure adequate caloric intake in older adults with impaired olfactory function $(14,15,18)$. Possibly these dietary adaptations to the loss of smell differ for smokers and non-smokers. Since we did not measure food intake or dietary choices, we cannot say whether this was the case in our cohort. Furthermore, central appetite regulation (e.g. by the secretion of hunger-hormone ghrelin and orexogenic neurons in the hypothalamus) is likely to safeguard adequate food intake, even in the absence of smell 
Table 2

The crude and adjusted associations of olfactory function with BMI, poor appetite and 3-year weight change in older participants of the Longitudinal Aging Study Amsterdam

\begin{tabular}{|c|c|c|c|}
\hline & B & OR 95\%-CI & P-value \\
\hline \multicolumn{4}{|c|}{ Association olfactory function - BMI } \\
\hline \multicolumn{4}{|l|}{ Smokers (n=96) } \\
\hline Crude model no. 1 & 0,225 & $0.071-0.379$ & $0.005 *$ \\
\hline Adjusted model no. $2^{\mathrm{a}}$ & 0,178 & $0.016-0.340$ & $0.032 *$ \\
\hline \multicolumn{4}{|c|}{ Association olfactory function - BMI } \\
\hline \multicolumn{4}{|l|}{ Non-smokers $(n=534)$} \\
\hline Crude model no. 1 & $-0,042$ & $-0,175$ & 0,341 \\
\hline Adjusted model no. $2^{\mathrm{a}}$ & $-0,015$ & $-0,172$ & 0,732 \\
\hline \multicolumn{4}{|c|}{ Association olfactory function-poor appetite } \\
\hline \multicolumn{4}{|l|}{ All participants $(n=673)$} \\
\hline Crude model no. 1 & 1,029 & $0.971-1.090$ & 0,337 \\
\hline Adjusted model no. $2^{\mathrm{b}}$ & 1,062 & $0.981-1.150$ & 0,137 \\
\hline \multicolumn{4}{|c|}{ Association olfactory function - prospective 3-year weight change } \\
\hline \multicolumn{4}{|l|}{ All participants $(\mathrm{n}=\mathbf{5 1 0})$} \\
\hline Crude model no. $1^{\mathrm{c}}$ & $-0,033$ & & 0,447 \\
\hline Adjusted model no. $2^{\mathrm{d}}$ & $-0,027$ & & 0,548 \\
\hline
\end{tabular}

(29). Nicotine, however, has been demonstrated to modulate the effects of ghrelin and promote anorexogenic neuron activation in the hypothalamus (30). With the modulation of central appetite regulation by nicotine, the relative influence of olfactory function on appetite and food intake could become more prominent in smokers than non-smokers. Additionally, our smokers had lower median [IQR] UPSIT-scores than our non-smokers (31 (28-34) versus 33 (31-35), respectively $(\mathrm{p}$-value $<0.001))$. The more severe olfactory dysfunction in smokers is likely to affect nutritional status to a greater extent than the milder olfactory dysfunction in non-smokers.

To evaluate the association between smoking and olfactory function further, we performed a post-hoc analysis in which we tested the differences in continuous UPSIT-score among current-, former-, and never smokers, using a Kruskall-Wallis test with Bonferroni correction. There was a significant difference in median UPSIT-score between never smokers and current smokers (33 versus 31, respectively, adjusted $\mathrm{p}=0.002$ ) and between former smokers and current smokers (33 versus 31 , respectively, adjusted $\mathrm{p}=0.001)$, but not between former smokers and never smokers (33 versus 33, adjusted $\mathrm{p}=1.000$ ). This is in line with the results of Murphy et al. (8), suggesting that the impact of smoking on olfactory function is reversible. We did not find the earlier reported dose-dependent relationship among smokers (31) as the number of cigarettes smoked per week did not differ among the olfactory function categories.

Our results show that olfactory function is not significantly associated with appetite. This is in line with Arganini et al. (17), but contrasts the findings of de Jong et al. (16). It is noteworthy that only the latter study assessed appetite by multiple questions instead of one. We cannot exclude that a single question may lack sensitivity in detecting poor appetite. However, poor appetite using this single question has been shown to be predictive of future incidence of malnutrition (1).

Our study is the first to investigate the association of olfactory function with prospective weight change. However, we did not find the two to be significantly associated. This might be due to the singular measurement of olfactory function. It is not known for how long any olfactory impairments existed and to what extent this had already influenced nutritional status. We cannot preclude that the onset of olfactory impairments was still recent in the relatively younger and more vital older adults in our cohort. Olfactory impairments may therefore not yet have impacted nutritional status (32). Contrarily, if olfactory impairments were more long-standing, they could have already caused food-intake and energy balance to shift towards a new equilibrium in which the participants' weight are stable.

The association we and others $(11,33)$ found between education and olfactory function scores could be explained by an advantage of higher educated participants in correctly 


\section{THE JOURNAL OF NUTRITION, HEALTH \& AGINGC}

carrying out a smell identification test. Consistent with literature $(8,11,17,18,20)$, self-awareness of impaired olfactory function in our study population was low with $38.5 \%$ of anosmic participants reporting no smell difficulties. Possibly, the underreporting 38.5\% represent the proportion of participants who have been anosmic for a long period of time. They might therefore not have recognized a decline in olfactory function. Still, these findings demonstrate the need for objective olfactory tests when olfactory dysfunction is suspected. There was also no difference in gender among olfactory function categories, which can be contributed to the gender-specific cutoffs for these groups. When using the Mann-Whitney U test to assess the difference in continuous UPSIT-score between men and women, women had higher UPSIT scores $(p=0.013)$, consistent with literature $(14,20,34,35)$.

There are several strengths to the present study. First, the study includes a large representative sample. Additionally, because LASA included data on many life domains, there was ample opportunity to correct for potential confounders. However, residual confounding can never be excluded. Third, olfactory function was objectively measured by a valid, extensive 40-item standardized test with high test-retest reliability ( $\mathrm{r}=0.918)(36)$.

A first limitation of the study is the potential selection bias. Out of 1023 LASA-participants, 199 declined taking the UPSIT-test. Of those taking the test, 151 had one or more missing items in the UPSIT-test and were excluded from the analyses. Testing the differences in characteristics between the included and excluded participants revealed that participants who declined taking the UPSIT-test or had incomplete tests were more likely to be smokers than those who had completed the UPSIT-test (29.5\% and $22.0 \%$ versus $15.1 \%$, respectively). They were also more likely to have a poor cognitive status (7.0\% and $5.3 \%$ versus $1.6 \%$, respectively) and a lower education level ( $12.1 \%$ and $19.2 \%$ versus $7.7 \%$, respectively). Both smoking and low education were associated with lower UPSIT-scores in our analytic sample. To investigate the potential impact of excluding those with incomplete tests, we performed multiple imputation on the missing UPSIT-items and repeated the regression analysis in a total of 767 older adults on UPSIT-score and BMI. The findings were similar to the current results. Again there was effect modification by smoking status $(\mathrm{p}=0.030)$, and again both the crude and adjusted model showed a significant positive association of UPSIT-score with BMI in smokers (adjusted regression model $\mathrm{B}=0.175, \mathrm{SE}=0.075$, $\mathrm{p}=0.020$ ), but not in non-smokers (adjusted regression model $\mathrm{B}=0.022, \mathrm{SE}=0.039, \mathrm{p}=0.547)$. These results suggest that potential selection bias did not affect the conclusion of our study.

A second limitation is that we only used an odor identification test and not an additional threshold test. It is possible that perceiving a scent rather than correctly identifying it, is more important in making food related choices. Therefore, using an identification test may have caused an underestimation of any association of olfactory function with nutritional status. It must be noted, however, that threshold tests are time-consuming and might be considered too tiresome (19). Furthermore, typically only one non-food odor is tested in threshold tests, which decreases its validity to objectify overall olfactory function in relation to nutrition.

A final limitation is the cross-sectional design of our study. Hence, we cannot assume a cause-effect relationship between olfactory function and BMI in smokers. Future prospective studies using repeated measurements of both olfactory function, dietary intake and nutritional status in the young-old and oldold are therefore needed.

In conclusion, lower olfactory function scores were associated with lower BMI in smokers, but not in non-smokers. Smoking older adults with poor olfactory function might therefore pose as a vulnerable group for the development of undernutrition. Lower olfactory scores were not associated with poorer appetite or prospective weight loss in our sample of community-dwelling Dutch older adults, aged 55-65 years. More prospective studies are needed in which different aspects of olfactory function are determined, including identification and threshold tests. Moreover, more data on nutritional status, such as body composition, dietary patterns, and food preferences need to be collected to elucidate the precise nature of the relationship between olfactory function and nutritional state in older persons.

\section{Conflict of interest: No competing interests declared.}

Funding: This study was supported by the European Union Horizon 2020 PROMISS project 'PRevention Of Malnutrition In Senior Subjects in the EU' (grant agreement no. 678732). The content only reflects the author's view and the Commission is not responsible for any use that may be made of the information it contains. MN is supported by a personal ZONMW-VIDI grant 2013 (grant agreement no. 016.146.327) and a Dutch Heart Foundation CVON IN CONTROL Young Talent Grant 2013. DR and HB are supported by a grant from the Dutch Parkinson Foundation.

Ethical Standards: This study complies with the current laws on research in the Netherlands.

Open Access: This article is distributed under the terms of the Creative Commons Attribution 4.0 International License (http://creativecommons.org/licenses/by/4.0/), which permits use, duplication, adaptation, distribution and reproduction in any medium or format, as long as you give appropriate credit to the original author(s) and the source, provide a link to the Creative Commons license and indicate if changes were made.

\section{References}

1. Schilp J, Kruizenga HM, Wijnhoven HA, Leistra E, Evers AM, van Binsbergen JJ, et al. High prevalence of undernutrition in Dutch community-dwelling older individuals. Nutrition. 2012;28(11-12):1151-6.

2. Lochs H, Allison SP, Meier R, Pirlich M, Kondrup J, Schneider S, et al. Introductory to the ESPEN Guidelines on Enteral Nutrition: Terminology, definitions and general topics. Clin Nutr. 2006;25(2):180-6.

3. Netherlands HCot. Undernutrition in the elderly. 2011

4. Garssen J. Demografie van de vergrijzing. Statistiek CBvd; 2011.

5. van der Pols-Vijlbrief R, Wijnhoven HA, Schaap LA, Terwee CB, Visser M. Determinants of protein-energy malnutrition in community-dwelling older adults: a systematic review of observational studies. Ageing Res Rev. 2014;18:112-31.

6. Wysokinski A, Sobow T, Kloszewska I, Kostka T. Mechanisms of the anorexia of aging-a review. Age (Dordr). 2015;37(4):9821.

7. Schiffman SS. Taste and smell losses in normal aging and disease. JAMA. 1997;278(16):1357-62.

8. Murphy C, Schubert CR, Cruickshanks KJ, Klein BE, Klein R, Nondahl DM. Prevalence of olfactory impairment in older adults. JAMA. 2002;288(18):2307-12.

9. Doty RL, Kamath V. The influences of age on olfaction: a review. Front Psychol 2014;5:20. 


\section{THE ASSOCIATION OF OLFACTORY FUNCTION WITH BMI, APPETITE, AND PROSPECTIVE WEIGHT CHANGE}

10. Doty RL, Shaman P, Applebaum SL, Giberson R, Siksorski L, Rosenberg L. Smell identification ability: changes with age. Science. 1984;226(4681):1441-3.

11. Seubert J, Laukka EJ, Rizzuto D, Hummel T, Fratiglioni L, Backman L, et al. Prevalence and Correlates of Olfactory Dysfunction in Old Age: A Population-Based Study. J Gerontol A Biol Sci Med Sci. 2017;72(8):1072-9.

12. Schiffman SS, Graham BG. Taste and smell perception affect appetite and immunity in the elderly. Eur J Clin Nutr. 2000;54 Suppl 3:S54-63.

13. Hummel T NS. Olfactory disorders and their consequences for quality of life. Acta Otolaryngol 2005;125(2):116-21

14. Gopinath B, Russell J, Sue CM, Flood VM, Burlutsky G, Mitchell P. Olfactory impairment in older adults is associated with poorer diet quality over 5 years. Eur J Nutr. 2016;55(3):1081-7.

15. Duffy VB, Backstrand JR, Ferris AM. Olfactory dysfunction and related nutritional risk in free-living, elderly women. J Am Diet Assoc. 1995;95(8):879-84; quiz 85-6.

16. de Jong N, Mulder I, de Graaf C, van Staveren WA. Impaired sensory functioning in elders: the relation with its potential determinants and nutritional intake. J Gerontol A Biol Sci Med Sci. 1999;54(8):B324-31.

17. Arganini C, Sinesio F. Chemosensory impairment does not diminish eating pleasure and appetite in independently living older adults. Maturitas. 2015;82(2):241-4

18. Smoliner C, Fischedick A, Sieber CC, Wirth R. Olfactory function and malnutrition in geriatric patients. J Gerontol A Biol Sci Med Sci. 2013;68(12):1582-8.

19. Toussaint N, de Roon M, van Campen JP, Kremer S, Boesveldt S. Loss of olfactory function and nutritional status in vital older adults and geriatric patients. Chem Senses 2015;40(3):197-203.

20. Karpa MJ, Gopinath B, Rochtchina E, Jie Jin W, Cumming RG, Sue CM, et al Prevalence and neurodegenerative or other associations with olfactory impairment in an older community. J Aging Health. 2010;22(2):154-68.

21. Huisman M, Poppelaars J, van der Horst M, Beekman AT, Brug J, van Tilburg TG, et al. Cohort profile: the Longitudinal Aging Study Amsterdam. Int J Epidemiol. 2011;40(4):868-76

22. Hoogendijk EO, Deeg DJ, Poppelaars J, van der Horst M, Broese van Groenou MI Comijs HC, et al. The Longitudinal Aging Study Amsterdam: cohort update 2016 and major findings. Eur J Epidemiol. 2016;31(9):927-45.

23. Doty RL, Shaman P, Kimmelman CP, Dann MS. University of Pennsylvania Smell Identification Test: a rapid quantitative olfactory function test for the clinic Laryngoscope. 1984;94(2 Pt 1):176-8.

24. Doty RL. The smell identification test administration Manual. 3. Haddon Heights, NJ:
Sensonics, Inc 1995.

25. Radloff L. The CES-D scale: a self-reported depression scale for research in the general population. Appl Psychol Measur. 1977;1:385-401.

26. Wijnhoven HA, Schilp J, van Bokhorst-de van der Schueren MA, de Vet HC, Kruizenga HM, Deeg DJ, et al. Development and validation of criteria for determining undernutrition in community-dwelling older men and women: The Short Nutritional Assessment Questionnaire 65+. Clin Nutr. 2012;31(3):351-8.

27. Garretsen HRF, Knibbe RA. Alcohol Prevalentie Onderzoek Rotterdam/ Limburg, Landelijk Eindrapport, Ministerie van Welzijn, Volksgezondheid en Cultuur, Leidschendam 1983.

28. Folstein MF, Folstein SE, McHugh PR. "Mini-mental state". A practical method for grading the cognitive state of patients for the clinician. J Psychiatr Res. 1975;12(3):189-98.

29. Morton GJ, Cummings DE, Baskin DG, Barsh GS, Schwartz MW. Central nervous system control of food intake and body weight. Nature. 2006;443(7109):289-95.

30. Hu T, Yang Z, Li MD. Pharmacological Effects and Regulatory Mechanisms of Tobacco Smoking Effects on Food Intake and Weight Control. J Neuroimmune Pharmacol. 2018.

31. Vennemann MM, Hummel T, Berger K. The association between smoking and smell and taste impairment in the general population. J Neurol. 2008;255:1121-6.

32. Zhang $\mathrm{C}$, Wang $\mathrm{X}$. Initiation of the age-related decline of odor identification in humans: A meta-analysis. Ageing Res Rev. 2017;40:45-50.

33. Boesveldt S, Lindau ST, McClintock MK, Hummel T, Lundstrom JN. Gustatory and olfactory dysfunction in older adults: a national probability study. Rhinology. 2011;49(3):324-30.

34. Gunzer W. Changes of Olfactory Performance during the Process of Aging Psychophysical Testing and Its Relevance in the Fight against Malnutrition. J Nut Health Aging. 2017;21(9):1010-5.

35. Dong J, Pinto JM, Guo X, Alonso A, Tranah G, Cauley JA, et al. The Prevalence of Anosmia and Associated Factors Among U.S. Black and White Older Adults. The Journals of Gerontology: Series A. 2017;72(8):1080-6.

36. Doty RL, Shaman P, Dann M. Development of the University of Pennsylvania Smell Identification Test: a standardized microencapsulated test of olfactory function. Physiol Behav. 1984;32(3):489-502. 